\title{
DIPTERA FLOWER VISITORS OF ADONIS VERNALIS IN THE BAKONY MTS (HUNGARY)
}

\author{
Tünde MÉszÁros ${ }^{1 *}$ and Sándor Tót $\mathrm{H}^{2}$ \\ ${ }^{1}$ Department of Plant Sciences and Biotechnology, Georgikon Faculty, University of Pannonia \\ H-8360 Keszthely, Festetics u. 7, Hungary; *meszarost773@gmail.com \\ ${ }^{2} \mathrm{H}-8420$ Zirc, Széchenyi u. 2, Hungary
}

Mészáros, T. \& Tóth, S. (2020): Diptera flower visitors of Adonis vernalis in the Bakony Mts (Hungary). - Studia bot. bung. 51(1): 41-56.

\begin{abstract}
The number of pollinators has decreased significantly in the last decades, producing a situation that is frequently mentioned as a "pollination crisis". In our study we document Diptera taxa collected on the early-flowering, legally protected Adonis vernalis L. According to our former observations, species of the Aculeata suborder (Hymenoptera) are the main pollinators of $A$. vernalis. Besides them many insects visit the flowers of Adonis, thus helping pollination directly or indirectly. Diptera taxa were collected in the spring of 2019 from three sites of the Bakonyvidék Mesoregion in Hungary. At Szentkirályszabadja and Veszprém-Kádárta villages the species Sphaerophoria scripta (19 and 10 individuals, respectively), on the Csatár Hill Chrysotoxum vernale and Pipizella viduata (3-3 individuals) were found the most frequent visitors. At each study site most of the collected insects were members of the Syrphidae family. Of the collected Diptera species only 2 species belonged to the Nematocera suborder, all the others belonged to the Brachycera suborder. At Veszprém-Kádárta we also found three Tephritidae larvae in the flowers.
\end{abstract}

Key words: flower-visiting insects, fly, protected plant species, Sphaerophoria, Syrphidae

\section{INTRODUCTION}

The number of pollinators has decreased significantly in the last decades, producing a situation that is frequently mentioned as a "pollination crisis". The crisis is partly due to the spread of agriculture at the expense of natural and seminatural grasslands, which considerably decreases the food resources of flower visitor and pollinator insects. This phenomenon is not only economically worrying, but has unfavourable effects on biodiversity and nature conservation as well (Allen-Wardell et al. 1998, Novais et al. 2016).

Diptera taxa have a significant role in many pollination systems and networks (KeARns 2002, KeVAN 2002, SsYMANK et al. 2008). They get protein from pollen, while flowers provide them shelter and can function as place of meeting or breeding (PAT Kó 2017). Sun facing flowers provide a shelter that is warmer than the surrounding air temperature. Compared to Hymenoptera species, Diptera taxa have less hair on their body, and generally they do not have special pollen 
collecting structures. Nevertheless, pollen can stick to their body, so they potentially can help pollination (KEARNS 2002, KEVAN 2002, SsYMANK et al. 2008).

The number of Diptera species is estimated at 400,000-800,000. In spite of this large number, the order is poorly studied taxonomically. 115 Diptera families occur in Hungary, and among them hoverflies are most thoroughly studied (Soltész 2017). Most Diptera species are restricted to habitats that provide optimal circumstances for them. The species composition of Diptera fauna in forests and grasslands is very different (ТóтH 1975).

Empididae species have strong proboscis. They are generally predatory flies hunting mainly for other fly species (cannibalism is frequent). Nonetheless, some of them are flower visitors feeding on nectar (Tóтн 1975).

Stratiomyidae adults can often be collected on flowers as they feed on nectar and pollen. Their larvae usually evolve in water, and the adults also prefer humid habitats like marshes (Tó TH 1975).

The larvae of Tephritidae live in different plant organs. Most of them evolve in Asteraceae species (Tót 1975).

Bombyliidae species are generally medium sized and often hairy. They feed on nectar. Some species hover in the air over the flowers and use their long, straight proboscis to reach the nectar. Other species land on flowers to feed (Tóth 1975).

Hoverflies play important role in food chains, since both their adults and larvae are food resources to predatory insects and other arthropods, birds, and vertebrates (Tó тн 2011). Adult hoverflies (Syrphidae) feed on nectar (Тóтн 1975), but they eat honeydew and sap leaking from wounded trees as well. Hoverflies feeding on pollen and/or nectar (similarly to bees and other flower visiting insects) can take part in pollination as they fly from flower to flower. However, the larvae of some hoverflies (e.g. Eumerus, Merodon) damage the bulb of plants.

According to our earlier observations species of the Aculeata suborder (Hymenoptera) are the main pollinators of $A$. vernalis (MÉszÁros and JózAN 2018). Besides them many insects are visiting the flowers of Adonis, thus helping pollination directly or indirectly.

Pollinators play a key role in the maintenance of genetic variability of $A$. vernalis, and in the survival of its populations (DENisow et al. 2014). The flowers of $A$. vernalis are nectarless, and thus provide only pollen as a reward for flower visitor insects (Denisow et al. 2014). The results of ChitTKA et al. (1999) show that nectarless species have fewer visitors than those producing nectars, even if they flower at the same time. Nectarless species develop other strategies to attract insects. Denisow and Wrzesień (2006) observed Diptera taxa on A. vernalis flowers, but they did not present the name of species. 
Entomologists often curious about which plants are visited regularly or occasionally by different hoverfly species. Similar studies have been done in Hungary too, mainly in the Bakony Mts. The results were published in the monograph of the hoverfly fauna of the Bakony Mts (То́тн 2001). Foraging of adult hoverflies had been observed on 446 plant taxa. Plants visited by hoverflies are very various. According to the above-mentioned studies in the Bakony Mts, the most plant species were visited by Eristalis tenax (Linnaeus, 1758) (observed on 242 plant taxa), Eristalis arbustorum (Linnaeus, 1758) (237 plant taxa), and Sphaerophoria scripta (Linnaeus, 1758) (218 plant taxa).

Previous studies in the Bakony Mts have detected the following hoverflies on the flowers of Adonis aestivalis (which is a close relative of $A$. vernalis): Cheilosia pagana (Meigen, 1822), Episyrphus balteatus (De Geer, 1776), Eristalinus aeneus (Scopoli, 1763), Eristalis arbustorum (Linnaeus, 1758), E. tenax (Linnaeus, 1758), Eupeodes corollae (Fabricius, 1794), Helophilus pendulus (Linnaeus, 1758), Melanostoma mellinum (Linnaeus, 1758), Platycheirus clypeatus (Meigen, 1822), P. europaeus Goeldlin de Tiefenau, Maibach et Speight, 1990, Scaeva pyrast$r i$ (Linnaeus, 1758), Sphaerophoria scripta (Linnaeus, 1758). On the flowers of A. vernalis the following species have been identified: Cheilosia grossa (Fallén, 1817), C. mutabilis (Fallén, 1817), C. pagana (Meigen, 1822), Eristalinus aeneus (Scopoli, 1763), E. sepulchralis (Linnaeus, 1758), Eristalis arbustorum (Linnaeus, 1758), E. pertinax (Scopoli, 1763), E. tenax (Linnaeus, 1758), Eupeodes corollae (Fabricius, 1794), Melanostoma mellinum (Linnaeus, 1758), M. scalare (Fabricius, 1794), Platycheirus albimanus (Fabricius, 1781), P. clypeatus (Meigen, 1822), P. europaeus Goeldlin de Tiefenau, Maibach et Speight, 1990, Sphaerophoria scripta (Linnaeus, 1758), Syrphus ribesii (Linnaeus, 1758), S. torvus Osten-Sacken, 1875, S. vitripennis Meigen, 1822.

In our study we document Diptera taxa collected on the early-flowering, legally protected Adonis vernalis $\mathrm{L}$.

\section{MATERIALS AND METHODS}

\section{Study species}

Adonis vernalis is a perennial, early-flowering member of the Ranunculaceae family. Its flowers are solitary and bisexual. The yellow, silky shining petals open to sunlight and have no nectaries (Anonymous 2000, Denisow et al. 2014, JANKOWSKA-B£ASZCZUK 1988). In suitable weather the first buds open at the end of March, and the flowering can last until May. The longitudinal growth of primary shoots is simultaneous with the opening of the first flowers, and the appearance and growth of secondary shoots start at the same time. The flowers of 
primary shoots open first at all times, the buds on secondary shoots open later. As a consequence, buds, flowers, and fruits can be seen simultaneously on the same individual (MÁTHÉ 1977).

\section{Collecting Diptera}

Diptera individuals were collected in the spring of 2019 from three sites of the Bakonyvidék Mesoregion in Hungary (DövéNYi 2010) (Table 1). At Szentkirályszabadja and Veszprém-Kádárta villages the insects were collected in a 23-hour long period, while on the Csatár Hill the total length of collecting was 22 hours.

Only insects found on $A$. vernalis flowers were collected. The sites were scanned by $1-3$ researchers continuously. Insects were collected with a butterfly net $30 \mathrm{~cm}$ in diameter, but the original net had been replaced by a dense and transparent tulle net.

Insects have been collected individually and put into glasses every hour. As a consequence, each individual has been counted once, representing a single flower visitation. For the sake of efficiency, the flies were not sorted according to their behaviour (e.g. feeding, sleeping, breeding in the flower). If larvae were found, they were put in glasses immediately.

The time of observation has been converted from standard time to summer time to make the comparison of data from different periods possible. The collected species have been identified by Sándor Tóth according to MAJER (1977), Mihályi (1960, 1975, 1979), Tóth (1977) and Wéber (1975).

\section{RESULTS}

At Szentkirályszabadja village we collected 91 individuals representing 36 species ( 8 families). $53.8 \%$ of individuals were male ( 49 individuals) and $46.2 \%$

Table 1. Adonis vernalis study sites.

\begin{tabular}{|c|c|c|c|c|c|c|}
\hline Settlement & $\mathrm{N}$ & $\mathrm{E}$ & $\begin{array}{l}\mathrm{Nr} \text { of in- } \\
\text { dividuals }\end{array}$ & $\begin{array}{c}\text { Habitat } \\
\text { type }\end{array}$ & $\begin{array}{c}\text { Study } \\
\text { area }\left(\mathrm{m}^{2}\right)\end{array}$ & $\begin{array}{l}\text { Collecting days } \\
\quad(2019)\end{array}$ \\
\hline $\begin{array}{l}\text { Szentkirály- } \\
\text { szabadja }\end{array}$ & 47.035700 & 17.950291 & ca 1,000 & $\begin{array}{l}\text { slope } \\
\text { steppe }\end{array}$ & 2,000 & $\begin{array}{c}\text { 30, } 31 \text { March, 4, } \\
\text { 6, } 19 \text { April }\end{array}$ \\
\hline Csatár Hill & 47.101894 & 17.853644 & $\begin{array}{c}20,000- \\
30,000\end{array}$ & $\begin{array}{c}\text { slope } \\
\text { steppe }\end{array}$ & 1,200 & $\begin{array}{c}\text { 19, } 30 \text { March, } \\
\text { 20, 21, } 30 \text { April, } \\
\text { 1, 2, } 3 \text { May }\end{array}$ \\
\hline $\begin{array}{l}\text { Veszprém- } \\
\text { Kádárta }\end{array}$ & 47.108191 & 17.956996 & ca 100 & $\begin{array}{l}\text { slope } \\
\text { steppe }\end{array}$ & 900 & $\begin{array}{c}15,17,22,24 \\
25,27,28 \text { April }\end{array}$ \\
\hline
\end{tabular}


female (42 individuals) (Tables 2-3). The lowest number of individuals were collected between 10-11 a.m. and 3-4 p.m. (6 individuals per hour), whereas most specimens were observed between 11 a.m.-1 p.m. and 2-3 p.m. (22 individuals per hour) (Fig. 1). At Veszprém-Kádárta village 49 individuals of 23 species (10 families) were collected. The ratio of males was $51 \%$ (25 individuals) and that of females $49 \%$ (24 individuals) (Tables $4-5$ ). The frequency of visitations was the lowest between 10-11 a.m. (3 individuals per hour) and the highest between 2-3 p.m. (17 individuals per hour) (Fig. 2). On the Csatár Hill we collected 24 individuals of 18 species ( 6 families). $29.2 \%$ of them was male (7 individuals) and $70.8 \%$ was female (17 individuals) (Tables 6-7). The lowest number of individuals were collected between 2-4 p.m. (0 individuals per hour), whereas most specimens were observed between 11-12 a.m. (12 individuals per hour) (Fig. 3). In sum, at the three sites the number of collected individuals was the lowest between 10-11 a.m. (14 individuals per hour), while the highest between 11-12 a.m. (41 individuals per hour) (Fig. 4).

At Szentkirályszabadja and Veszprém-Kádárta villages Sphaerophoria scripta (Linnaeus, 1758) was found the most frequent species (19 and 10 individuals), while on the Csatár Hill the species Chrysotoxum vernale Loew, 1841 and Pipizella viduata (Linnaeus, 1758) dominated with 3-3 individuals. At each site most of the collected insects were members of the Syrphidae family, only 2 belonged to the Nematocera suborder. All the others belonged to the Brachycera suborder. At Veszprém-Kádárta we also found three Tephritidae larvae in the flowers.

\section{DISCUSSION}

All Syrphidae species collected in our study had already been reported from the Bakony Mts, but not each of them had been observed on Adonis vernalis flowers. То́тн (2001) recognised 7 hoverfly species (Eupeodes corollae, Melanostoma mellinum, Platycheirus albimanus, Sphaerophoria scripta, Syrphus ribesii, S. torvus, S. vitripennis) on A. vernalis. We have recorded 24 Syrphidae species, so the list of hoverflies visiting $A$. vernalis increased by 17 new species. In contrast to former observations in the Bakony Mts, we have not noticed individuals of the following species on $A$. vernalis flowers: Cheilosia grossa (Fallén, 1817), C. mutabilis (Fallén, 1817), C. pagana (Meigen, 1822), Eristalinus aeneus (Scopoli, 1763), E. sepulchralis (Linnaeus, 1758), Eristalis arbustorum (Linnaeus, 1758), E. pertinax (Scopoli, 1763), E. tenax (Linnaeus, 1758), Melanostoma scalare (Fabricius, 1794), Platycheirus clypeatus (Meigen, 1822) and P. europaeus Goeldlin de Tiefenau, Maibach et Speight, 1990. 
Table 2. Adonis vernalis flower visitor Diptera species listed in decreasing frequency (Szentkirályszabadja, 2019).

\begin{tabular}{|c|c|c|c|c|c|}
\hline Species & Family & $\begin{array}{l}\text { Nr of in- } \\
\text { dividuals }\end{array}$ & Male & Female & $\begin{array}{c}\text { Time of } \\
\text { visitation }\end{array}$ \\
\hline $\begin{array}{l}\text { Sphaerophoria scripta (Linnaeus, } \\
1758 \text { ) }\end{array}$ & Syrphidae & 19 & 10 & 9 & $\begin{array}{l}10 \text { a.m. }-3 \\
\text { p.m. }\end{array}$ \\
\hline Syrphus vitripennis Meigen, 1822 & Syrphidae & 6 & 1 & 5 & $\begin{array}{l}12-1 \text { p.m., } \\
2-3 \text { p.m. }\end{array}$ \\
\hline Brachypalpus valgus (Panzer, 1798) & Syrphidae & 4 & 3 & 1 & $12-1$ p.m. \\
\hline Eupeodes corollae (Fabricius, 1794) & Syrphidae & 4 & 1 & 3 & $1-3$ p.m. \\
\hline Myathropa florea (Linnaeus, 1758) & Syrphidae & 4 & 1 & 3 & $\begin{array}{l}11 \text { a.m. }-1 \\
\text { p.m. }\end{array}$ \\
\hline Sepsis punctum (Fabricius, 1794) & Sepsidae & 4 & 3 & 1 & $11-12$ a.m. \\
\hline Syrphus torvus Osten-Sacken, 1875 & Syrphidae & 4 & 2 & 2 & $\begin{array}{l}10-12 \text { a.m., } \\
1-2 \text { p.m. }\end{array}$ \\
\hline Anthomyidae sp. indet. & Anthomyidae & 3 & 1 & 2 & $\begin{array}{l}11-12 \text { a.m., } \\
1-2 \text { p.m. }\end{array}$ \\
\hline Delia radicum (Linnaeus, 1758) & Anthomyidae & 3 & 2 & 1 & 2-3 p.m. \\
\hline $\begin{array}{l}\text { Melanostoma mellinum (Linnaeus, } \\
\text { 1758) }\end{array}$ & Syrphidae & 3 & 2 & 1 & $11-12$ a.m. \\
\hline $\begin{array}{l}\text { Meroplius minutus (Widemann, } \\
1830 \text { ) }\end{array}$ & Sepsidae & 3 & 1 & 2 & $11-12$ a.m. \\
\hline $\begin{array}{l}\text { Neoascia podagrica (Fabricius, } \\
1775 \text { ) }\end{array}$ & Syrphidae & 3 & 2 & 1 & $1-2$ p.m. \\
\hline $\begin{array}{l}\text { Syritta pipiens Le Peletier et Ser- } \\
\text { ville, } 1828\end{array}$ & Syrphidae & 3 & 1 & 2 & $11-12$ a.m. \\
\hline Syrphus ribesii (Linnaeus, 1758) & Syrphidae & 3 & 2 & 1 & $\begin{array}{l}\text { 10-11 a.m., } \\
2-3 \text { p.m. }\end{array}$ \\
\hline Delia cardui (Meigen, 1826) & Anthomyidae & 2 & 2 & & 3-4 p.m. \\
\hline Gonia ornata Meigen, 1826 & Tachinidae & 2 & 1 & 1 & $12-1$ p.m. \\
\hline $\begin{array}{l}\text { Platycheirus albimanus (Fabricius, } \\
\text { 1781) }\end{array}$ & Syrphidae & 2 & 2 & & $12-1$ p.m. \\
\hline Anthomyia imbrida Rondani, 1866 & Anthomyidae & 1 & 1 & & $3-4$ p.m. \\
\hline Carcelia sp. indet. & Tachinidae & 1 & & 1 & $12-1$ p.m. \\
\hline Catharosia pygmaea (Fallén, 1815) & Tachinidae & 1 & 1 & & $12-1$ p.m. \\
\hline $\begin{array}{l}\text { Conophorus virescens (Fabricius, } \\
1787 \text { ) }\end{array}$ & Bombyliidae & 1 & & 1 & $2-3$ p.m. \\
\hline Culex pipiens pipiens Linnaeus, 1758 & Culicidae & 1 & 1 & & 3-4 p.m. \\
\hline $\begin{array}{l}\text { Dasysyrphus venustus (Meigen, } \\
1822 \text { ) }\end{array}$ & Syrphidae & 1 & 1 & & $10-11$ a.m. \\
\hline
\end{tabular}


Table 2. (continued)

\begin{tabular}{|c|c|c|c|c|c|}
\hline Species & Family & $\begin{array}{l}\mathrm{Nr} \text { of in- } \\
\text { dividuals }\end{array}$ & Male & Female & $\begin{array}{c}\text { Time of } \\
\text { visitation }\end{array}$ \\
\hline Empis opaca Meigen, 1904 & Empididae & 1 & 1 & & $11-12$ a.m. \\
\hline Epistrophe eligans (Harris, 1780) & Syrphidae & 1 & 1 & & 3-4 p.m. \\
\hline $\begin{array}{l}\text { Epistrophe melanostoma (Zetter- } \\
\text { stedt, 1843) }\end{array}$ & Syrphidae & 1 & & 1 & 3-4 p.m. \\
\hline Exorista larvarum (Linnaeus, 1758) & Tachinidae & 1 & 1 & & 2-3 p.m. \\
\hline Gonia divisa Meigen, 1826 & Tachinidae & 1 & & 1 & $1-2$ p.m. \\
\hline $\begin{array}{l}\text { Hebia flavipes Robineau-Desvoidy, } \\
1830\end{array}$ & Tachinidae & 1 & 1 & & $1-2$ p.m. \\
\hline Leucophora personata (Collin, 1922) & Anthomyidae & 1 & 1 & & 1-2 p.m. \\
\hline $\begin{array}{l}\text { Meliscaeva cinctella (Zetterstedt, } \\
1843 \text { ) }\end{array}$ & Syrphidae & 1 & & 1 & 2-3 p.m. \\
\hline Pollenia sp. indet. & Calliphoridae & 1 & & 1 & $10-11$ a.m. \\
\hline $\begin{array}{l}\text { Rhamphomyia crassicornis Fallén, } \\
\text { 1816) }\end{array}$ & Empididae & 1 & 1 & & $11-12$ a.m. \\
\hline $\begin{array}{l}\text { Sphaerophoria taeniata (Meigen, } \\
1822 \text { ) }\end{array}$ & Syrphidae & 1 & 1 & & 2-3 p.m. \\
\hline Tachina fera (Linnaeus, 1761) & Tachinidae & 1 & & 1 & $2-3$ p.m. \\
\hline Tachina lurida (Fabricius, 1781) & Tachinidae & 1 & 1 & & 10-11 a.m. \\
\hline Total & & $\begin{array}{c}91(36 \\
\text { species) }\end{array}$ & 49 & 42 & $\begin{array}{c}10 \text { a.m. }-4 \\
\text { p.m. }\end{array}$ \\
\hline
\end{tabular}

Table 3. Adonis vernalis flower visitor Diptera families listed in decreasing frequency (Szentkirályszabadja, 2019).

\begin{tabular}{lcccc}
\hline Family & Nr of individuals & Male & Female & Time of visitation \\
\hline Syrphidae & 60 & 30 & 30 & 10 a.m.-4 p.m. \\
Anthomyidae & 10 & 7 & 3 & $11-12$ a.m., $1-4$ p.m. \\
Tachinidae & 9 & 5 & 4 & $10-11$ a.m., $12-3$ p.m. \\
Sepsidae & 7 & 4 & 3 & $11-12$ a.m. \\
Empididae & 2 & 2 & & $11-12$ a.m. \\
Bombyliidae & 1 & & 1 & $2-3$ p.m. \\
Calliphoridae & 1 & & 1 & $10-11$ a.m. \\
Culicidae & 1 & 1 & & $3-4$ p.m. \\
\hline Total & 91 & 49 & 42 & 10 a.m.-4 p.m. \\
\hline
\end{tabular}


Table 4. Adonis vernalis flower visitor Diptera species listed in decreasing frequency (Veszprém-Kádárta, 2019).

\begin{tabular}{|c|c|c|c|c|c|}
\hline Species & Family & $\begin{array}{l}\text { Nr of in- } \\
\text { dividuals }\end{array}$ & Male & Female & $\begin{array}{c}\text { Time of } \\
\text { visitation }\end{array}$ \\
\hline $\begin{array}{l}\text { Sphaerophoria scripta (Linnae- } \\
\text { us, 1758) }\end{array}$ & Syrphidae & 10 & 8 & 2 & $1-3$ p.m. \\
\hline Tachina ursina Meigen, 1824 & Tachinidae & 4 & 2 & 2 & $\begin{array}{l}12-1 \text { p.m., } \\
2-3 \text { p.m. }\end{array}$ \\
\hline Empis chioptera Meigen, 1804 & Empididae & 4 & & 4 & $2-4$ p.m. \\
\hline Anthomyidae sp. indet. & Anthomyidae & 4 & 3 & 1 & 3-4 p.m. \\
\hline Syrphus ribesii (Linnaeus, 1758) & Syrphidae & 3 & 3 & & $\begin{array}{l}11-12 \text { a.m., } \\
2-3 \text { p.m. }\end{array}$ \\
\hline $\begin{array}{l}\text { Rhamphomyia atra Meigen, } \\
1822\end{array}$ & Empididae & 3 & 1 & 2 & $10-11$ a.m. \\
\hline $\begin{array}{l}\text { Syrphus vitripennis Meigen, } \\
1822\end{array}$ & Syrphidae & 2 & 1 & 1 & 2-3 p.m. \\
\hline Gonia ornata Meigen, 1826 & Tachinidae & 2 & 1 & 1 & 2-3 p.m. \\
\hline Empis femorata Fabricius, 1798 & Empididae & 2 & 1 & 1 & 3-4 p.m. \\
\hline Atylostoma tricolor (Mik, 1884) & Tachinidae & 2 & 1 & 1 & 12-1 p.m. \\
\hline $\begin{array}{l}\text { Sepsis punctum (Fabricius, } \\
1794 \text { ) }\end{array}$ & Sepsidae & 1 & 1 & & $11-12$ a.m. \\
\hline $\begin{array}{l}\text { Platystoma seminationis (Fabri- } \\
\text { cius, 1775) }\end{array}$ & Platystomatidae & 1 & & 1 & 3-4 p.m. \\
\hline $\begin{array}{l}\text { Oxyna parietina (Linnaeus, } \\
1758 \text { ) }\end{array}$ & Tephritidae & 1 & & 1 & $11-12$ a.m. \\
\hline Oxyna flavipennis (Loew, 1844) & Tephritidae & 1 & & 1 & $11-12$ a.m. \\
\hline $\begin{array}{l}\text { Limnophora tigrina Am Stein, } \\
1860\end{array}$ & Muscidae & 1 & & 1 & 2-3 p.m. \\
\hline $\begin{array}{l}\text { Hemipenthes morio (Linnaeus, } \\
1758 \text { ) }\end{array}$ & Bombyliidae & 1 & & 1 & 2-3 p.m. \\
\hline $\begin{array}{l}\text { Eupeodes corollae (Fabricius, } \\
1794 \text { ) }\end{array}$ & Syrphidae & 1 & & 1 & $11-12$ a.m. \\
\hline Empis albicans Meigen, 1822 & Empididae & 1 & 1 & & 2-3 p.m. \\
\hline Empididae sp. indet. & Empididae & 1 & & 1 & 2-3 p.m. \\
\hline $\begin{array}{l}\text { Chrysotoxum verralli Collin, } \\
1940\end{array}$ & Syrphidae & 1 & & 1 & $11-12$ a.m. \\
\hline $\begin{array}{l}\text { Chrysotoxum vernale Loew, } \\
1841\end{array}$ & Syrphidae & 1 & 1 & & $11-12$ a.m. \\
\hline $\begin{array}{l}\text { Carcelia tibialis (Robineau- } \\
\text { Desvoidy, 1863) }\end{array}$ & Tachinidae & 1 & 1 & & 3-4 p.m. \\
\hline Actina chalybea (Meigen, 1804) & Stratiomyidae & 1 & & 1 & 2-3 p.m. \\
\hline Total & & $\begin{array}{c}49(23 \\
\text { species })\end{array}$ & 25 & 24 & $\begin{array}{l}10 \text { a.m. }-4 \\
\text { p.m. }\end{array}$ \\
\hline
\end{tabular}


Table 5. Adonis vernalis flower visitor Diptera families listed in decreasing frequency (Veszprém-Kádárta, 2019).

\begin{tabular}{lcccc}
\hline Family & Nr of individuals & Male & Female & Time of visitation \\
\hline Syrphidae & 18 & 13 & 5 & $11-12$ a.m., 1-3 p.m. \\
Empididae & 11 & 3 & 8 & $10-11$ a.m., 2-4 p.m. \\
Tachinidae & 9 & 5 & 4 & $12-1$ p.m., 2-4 p.m. \\
Anthomyidae & 4 & 3 & 1 & $3-4$ p.m. \\
Tephritidae & 2 & & 2 & $11-12$ a.m. \\
Bombyliidae & 1 & & 1 & $2-3$ p.m. \\
Muscidae & 1 & & 1 & $2-3$ p.m. \\
Platystomatidae & 1 & 1 & & $3-4$ p.m. \\
Sepsidae & 1 & & 1 & $11-12$ a.m. \\
Stratiomyidae & 1 & 25 & 24 & 10 a.m.-4 p.m. \\
\hline Total & 49 & & &
\end{tabular}

* $=3$ larvae were found as well

Although the total length of collecting was similar at each site $(23,23$ and 22 hours), the number of both individuals and species differed significantly. The study site at Szentkirályszabadja village appeared to be the most species rich, and the highest number of individuals was recorded here ( 91 individuals of 36 species). It may be the consequence of the fact that this area is bordered by forests

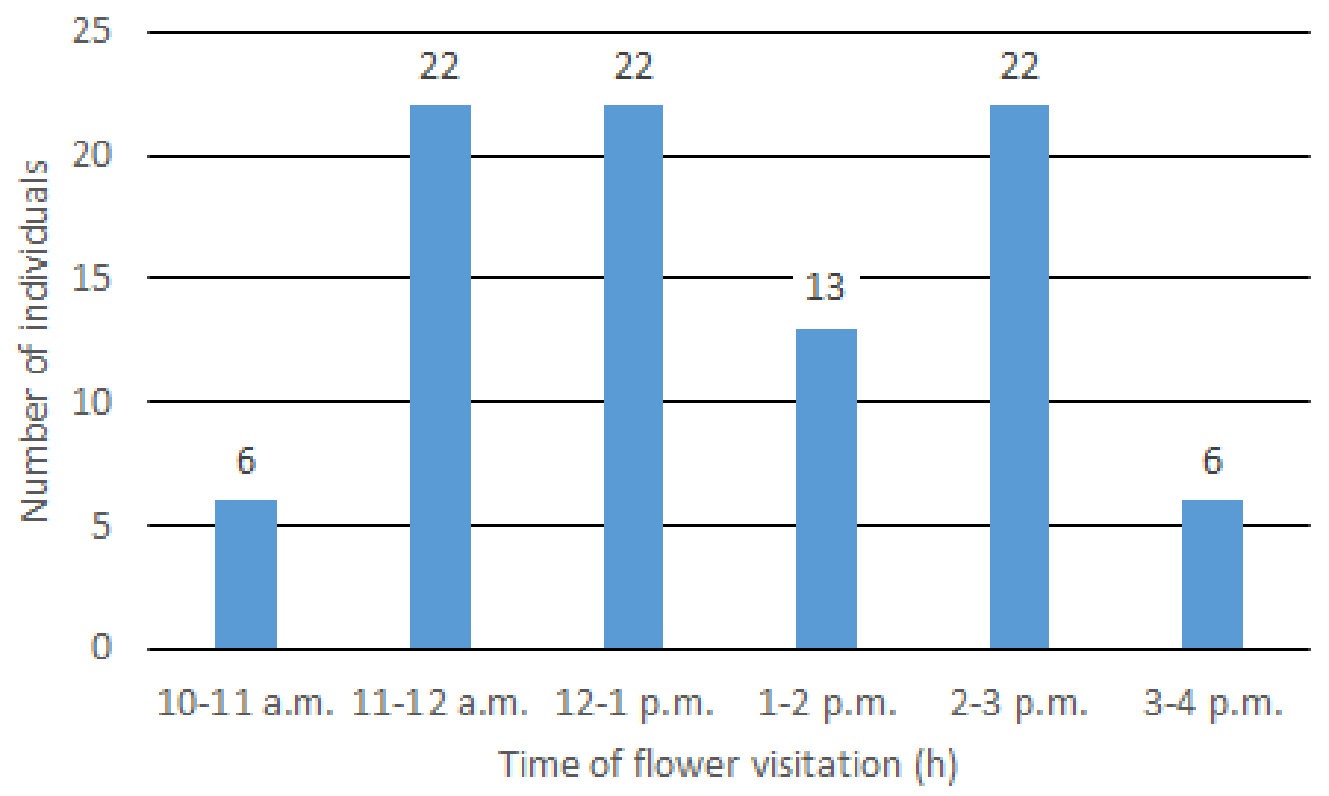

Fig. 1. Temporal distribution of Adonis vernalis flower visitor Diptera taxa at Szentkirályszabadja (2019). 


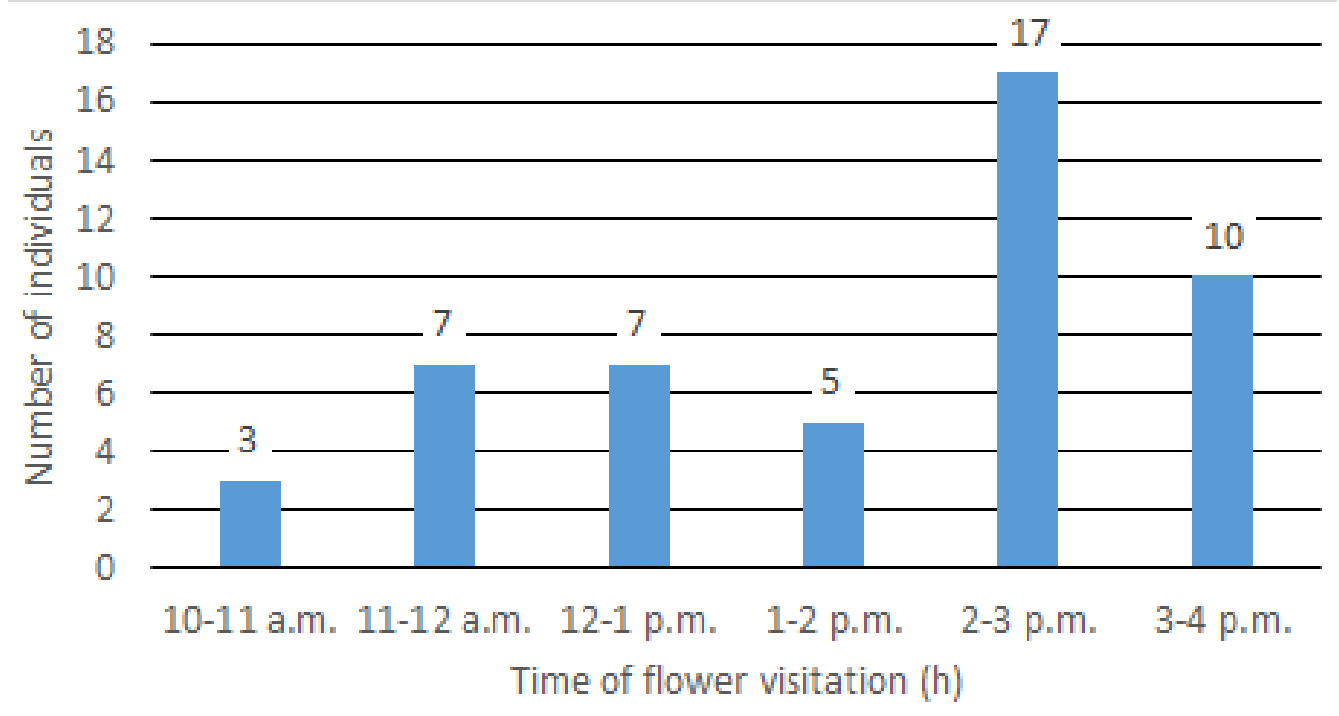

Fig. 2. Temporal distribution of Adonis vernalis flower visitor Diptera taxa at Veszprém-Kádárta (2019).

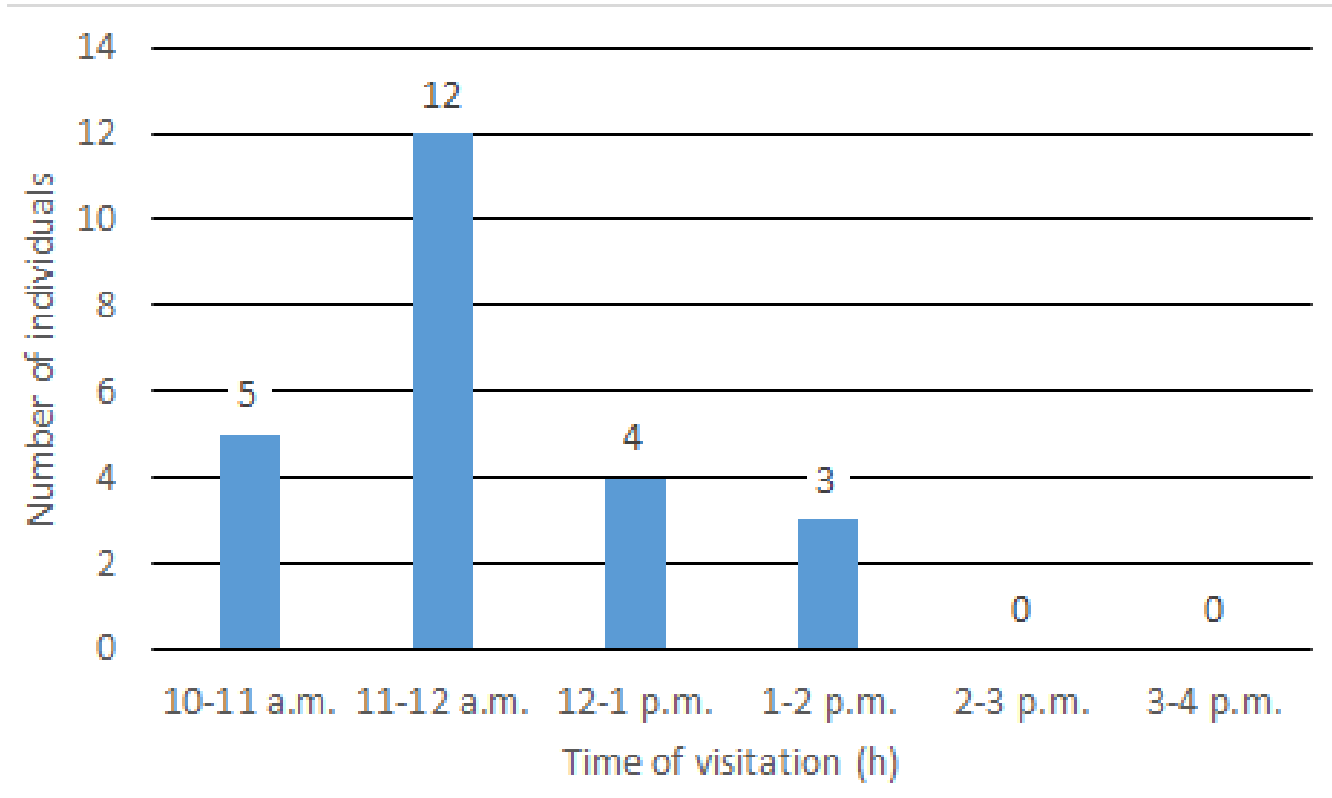

Fig. 3. Temporal distribution of Adonis vernalis flower visitor Diptera taxa on the Csatár Hill (2019). 


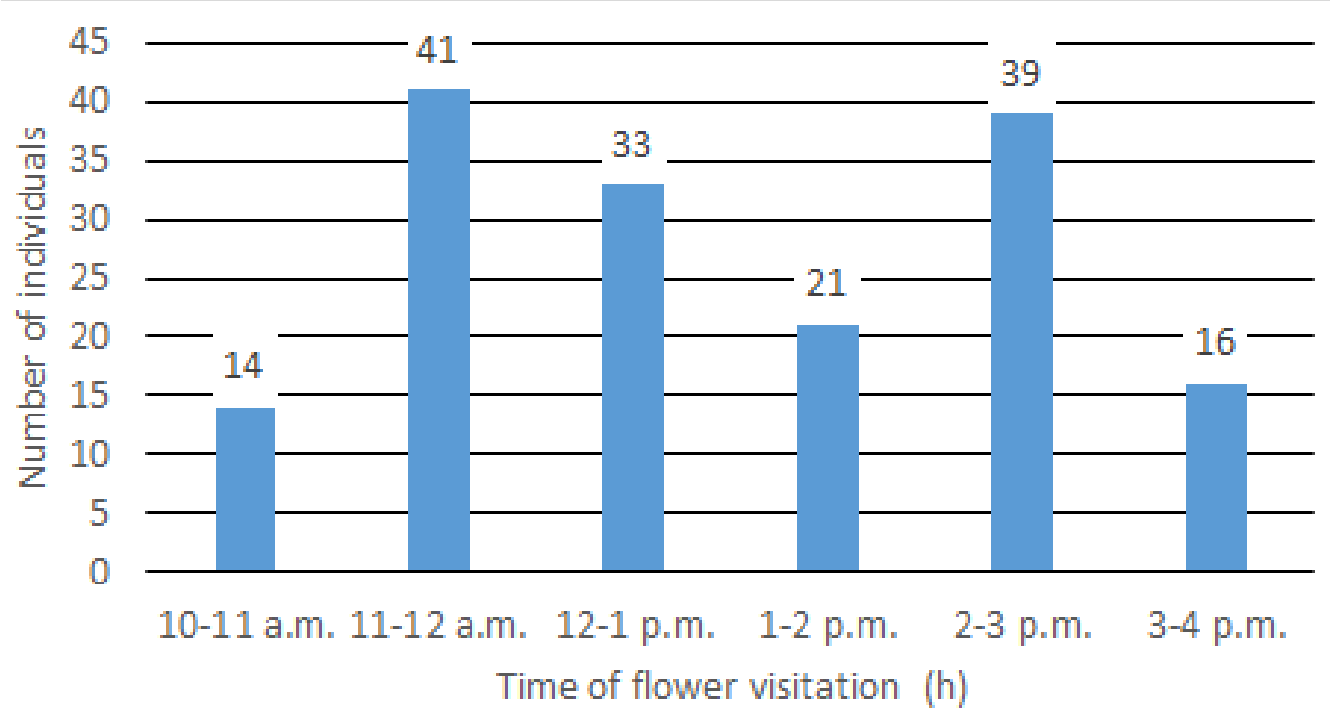

Fig. 4. Temporal distribution of Adonis vernalis flower visitor Diptera taxa at Szentkirályszabadja, Veszprém-Kádárta and on the Csatár Hill (2019).

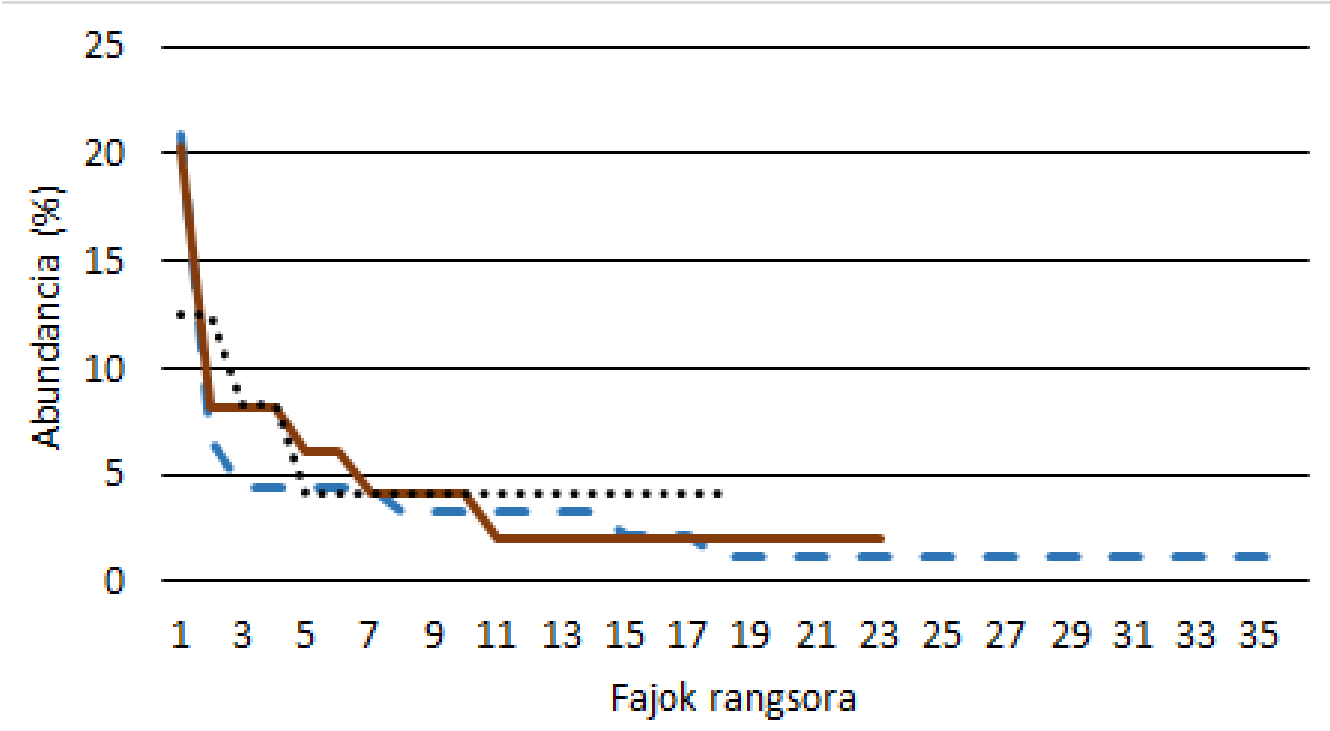

- Szentkirályszabadja - Veszprém-Kádárta ...... Csatár-hegy

Fig. 5. Rank abundance of Adonis vernalis flower visitor Diptera species. 
Table 6. Adonis vernalis flower visitor Diptera species listed in decreasing frequency (Csatár Hill, 2019).

\begin{tabular}{|c|c|c|c|c|c|}
\hline Species & Family & $\begin{array}{l}\text { Nr of indi- } \\
\text { viduals }\end{array}$ & Male & Female & $\begin{array}{l}\text { Time of } \\
\text { visitation }\end{array}$ \\
\hline Chrysotoxum vernale Loew, 1841 & Syrphidae & 3 & 1 & 2 & $\begin{array}{l}11 \text { a.m. }-1 \\
\text { p.m. }\end{array}$ \\
\hline Pipizella viduata (Linnaeus, 1758) & Syrphidae & 3 & 1 & 2 & $\begin{array}{l}\text { 10-11 a.m., } \\
1-2 \text { p.m. }\end{array}$ \\
\hline Delia sp. indet. & Anthomyidae & 2 & 1 & 1 & $11-12$ a.m. \\
\hline Empis albicans Meigen, 1822 & Empididae & 2 & 1 & 1 & $11-12$ a.m. \\
\hline Anthomyia pluvialis (Linnaeus, 1758) & Anthomyidae & 1 & & 1 & $1-2$ p.m. \\
\hline Empis chioptera Meigen, 1804 & Empididae & 1 & & 1 & $11-12$ a.m. \\
\hline $\begin{array}{l}\text { Epistrophe diaphana (Zetterstedt, } \\
\text { 1843) }\end{array}$ & Syrphidae & 1 & & 1 & $11-12$ a.m. \\
\hline Episyrphus balteatus (De Geer, 1776) & Syrphidae & 1 & & 1 & $1-2$ p.m. \\
\hline Exorista larvarum (Linnaeus, 1758) & Tachinidae & 1 & & 1 & $10-11$ a.m. \\
\hline Exorista tubulosa Herting, 1967 & Tachinidae & 1 & 1 & & $11-12$ a.m. \\
\hline $\begin{array}{l}\text { Pipizella divicoi (Goeldlin de Tiefe- } \\
\text { nau, 1974) }\end{array}$ & Syrphidae & 1 & 1 & & $10-11$ a.m. \\
\hline Sphaerophoria scripta (Linnaeus, 1758) & Syrphidae & 1 & & 1 & $12-1$ p.m. \\
\hline Tachinidae sp. indet. & Tachinidae & 1 & & 1 & $10-11$ a.m. \\
\hline $\begin{array}{l}\text { Xanthogramma laetum (Fabricius, } \\
1794 \text { ) }\end{array}$ & Syrphidae & 1 & & 1 & $11-12$ a.m. \\
\hline Sphaerophoria loewi Zetterstedt, 1843 & Syrphidae & 1 & & 1 & $11-12$ a.m. \\
\hline Empis livida Linnaeus, 1758 & Empididae & 1 & & 1 & 12-1 p.m. \\
\hline Aedes cinereus Meigen, 1818 & Culicidae & 1 & 1 & & $11-12$ a.m. \\
\hline Oxyna parietina (Linnaeus, 1758) & Tephritidae & 1 & & 1 & $11-12$ a.m. \\
\hline Total & & $\begin{array}{c}24(18 \\
\text { species })\end{array}$ & 7 & 17 & $\begin{array}{l}10 \text { a.m. }-2 \\
\text { p.m. }\end{array}$ \\
\hline
\end{tabular}

Table 7. Adonis vernalis flower visitor Diptera families listed in decreasing frequency (Csatár Hill, 2019).

\begin{tabular}{lcccc}
\hline Family & Nr of individuals & Male & Female & Time of visitation \\
\hline Syrphidae & 12 & 3 & 9 & 10 a.m.-2 p.m. \\
Empididae & 4 & 1 & 3 & 11 a.m.-1 p.m. \\
Anthomyidae & 3 & 1 & 2 & $11-12$ a.m., $1-2$ p.m. \\
Tachinidae & 3 & 1 & 2 & $10-12$ a.m. \\
Culicidae & 1 & 1 & & $11-12$ a.m. \\
Tephritidae & 1 & & 1 & $11-12$ a.m. \\
\hline Total & 24 & 7 & 17 & 10 a.m.-2 p.m. \\
\hline
\end{tabular}


that provide proper circumstances for Diptera. We collected the lowest number of individuals and species on the Csatár Hill. It can probably be explained by the surrounding orchards which function as pollen sources when A. vernalis is in bloom, thus they offer a feeding option for Diptera. Edge effect can be another factor because the area is located next to a road, and dust pollution, air movements and sound effects caused by traffic can have negative effects on Diptera. The study site on the Csatár Hill is situated far away from forests, what can also be a reason for the lowest number of individuals and species. According to our experiences, this area was windier in warm, sunny weather, while the other two sites were specifically warm and windless.

In Szentkirályszabadja and Veszprém-Kádárta Sphaerophoria scripta was the most frequent species, but on the Csatár Hill only one individual was found. According to previous Hungarian studies this species plays a prominent role in pollination. It is a widespread and frequent in Hungary. It is a typical xerothermic species of open areas, mainly occurring in dry grasslands, and active around midday. During the studies in the Bakony Mts it has been collected on Adonis vernalis as well. It was formerly known from Szentkirályszabadja as Sándor Tóth collected 1 male and 5 females on 27.06.1999 (То́тн 2001, 2011). The rank abundance curves (Fig. 5) show that in Szentkirályszabadja and Veszprém-Kádárta one species (Sphaerophoria scripta) was dominant, the number of collected individuals of other species was much lower. The two curves decrease sharply: at Szentkirályszabadja the dominant Sphaerophoria scripta (20.9\%) was followed by Syrphus vitripennis Meigen, 1822 (representing 6.6\%), while in VeszprémKádárta Sphaerophoria scripta (20.4\%) was followed by Tachina ursina Meigen, 1824 and Empis chioptera Meigen, 1804 (both reaching 8.2\%). The first section of the curve constructed at the Csatár Hill site decrease slightly because no species was dominant, 3, 2 and 1 individuals were collected from every species (12.5\%, $8.3 \%$ and $4.2 \%$ ). The last section of all of the three curves reached a plateau. This section proved to be the longest at Szentkirályszabadja site, because we collected 1 individual from most species (14) here.

Both individuals of collected Culicidae species were males, who feed on plant liquid (Tóth 1975). As $A$. vernalis is a nectarless species, these insects visited the flower for other services (shelter, warming, etc.), which shows the presence of combined plant functions.

Although the study of Tótн (1975) showed that adult Stratiomyidae live in rather humid habitats, we have found them in dry grasslands, too.

The three collected Tephritidae larvae also prove that flowers have many different and combined functions, and flowers can serve as breeding or growing places for insects in different periods of their life-cycle. 
The ratio of males and females was nearly equal at Veszprém-Kádárta. At Szentkirályszabadja the number of males was higher, while on the Csatár Hill more females were found. These results show that there is no significant difference in the flower visiting behaviour of males and females.

The temporal distribution of flower visitations was also different at the three sites. At Veszprém-Kádárta the period between 2-4 p.m. was the most effective (Fig. 2), while on the Csatár Hill no individuals were collected in this period. At the latter site insects were most active between 11-12 a.m. (Fig. 3). At Szentkirályszabadja the temporal distribution was more balanced between 11 a.m. and 3 p.m. The number of flower visitations decreased only between 1-2 p.m. (Fig. 1). If we analyse the data collected on the three sites together, we can see that the temporal distribution of flower visitations had peaks before mid-day (11-12 a.m.) and in early afternoon (2-3 p.m.) (Fig. 4).

Adonis vernalis offers forage (pollen) for insects already in early spring. The food resources provided by $A$. vernalis are extremely valuable because in the early period of year the number of flowering plants is limited. Diptera can carry pollen to stigmas as they move in flowers, so they can take part in pollination as well. Besides forage flowers provide other services as well, such as shelter, place of rest, breeding, warming, larval evolution. These functions are very important for Diptera and help maintain biodiversity. Our results are contributions to the knowledge of ecology of $A$. vernalis, and provide data about flower visiting behaviour of Diptera in general.

Acknowledgements - We would like to thank Dénes Péteri and Gábor Barad for their help in insect collection. We would like to thank Judit Bódis for her comments on the manuscript. The publication is supported by the EFOP-3.6.3-VEKOP-16-2017-00008 project. The project is co-financed by the European Union and the European Social Fund.

Összefoglaló: Az utóbbi évtizedekben a megporzó rovarok száma jelentősen visszaesett, így a napjainkban sokat emlegetett „pollinációs krízis” nyilvánvalóvá vált. Tanulmányunkban a kora tavaszi virágzású, védett Adonis vernalis L. virágokon gyüjtött kétszárnyúakat (Diptera) ismertetjük. Korábbi megfigyeléseink alapján az A. vernalis fö megporzói az Aculeata alrendből kerülnek ki. Az Aculeata fajokon kívül azonban számos rovar látogatja a virágokat, melyek közvetlenül vagy közvetve hozzájárulhatnak a megporzáshoz. A gyüjtéseket 2019 tavaszán végeztük három területen, melyek földrajzilag a Bakonyvidék középtáj területén helyezkednek el. Szentkirályszabadján és Veszprém-Kádártán Sphaerophoria scripta (Linnaeus, 1758) egyedből gyüjtöttük a legtöbbet (19 és 10 egyed), a Csatár-hegyen pedig Chrysotoxum vernale Loew, 1841 és Pipizella viduata (Linnaeus, 1758) egyedekből (3-3 egyed). A legtöbb egyed mindhárom területen a zengőlegyekből (Syrphidae) került ki. Az összes gyüjtött Diptera egyedből csupán 2 egyed tartozott a Nematocera alrendbe, a többi egyed a Brachycera alrendet képviselte. Veszprém-Kádártán három Tephritidae lárvát is találtunk a virágokban. 


\section{REFERENCES}

Allen-Wardell, G., Bernhardt, P., Bitner, R., Burquez, A., Buchmann, S., Cane, J., Cox, P. A., Dalton, V., Feinsinger, P., Ingram, M., Inouye D., Jones, C. E., Kennedy, K., Kevan, P., Koopowitz, H., Medellin, R., Medellin-Morales, S., Nabhan, G. P., Pavlik, B., Tepedino, V., Torchio, P. and Walker, S. (1998): The potential consequences of pollinator declines on the conservation of biodiversity and stability of food crop yields. Conservation Biology 12: 8-17. https://doi.org/10.1111/j.1523-1739.1998.97154.x

Anonymous (2000): Inclusion of Adonis vernalis in Appendix II in accordance with Article II 2(a). https://cites.org/sites/default/fi les/eng/cop/11/prop/61.pdf. (accessed: 05.08.2019)

Chitt ka, L., Thomson, J. D. and Waser, N. M. (1999): Flower constancy, insect psychology, and plant evolution. - Naturwiss. 86 (8): 361-377.

Denisow, B. and Wrzesień, M. (2006): The study of blooming and pollen efficiency of Adonis vernalis L. in xerothermic plant communities. - J. Apicult. Sci. 50(1): 25-32.

Denisow, B., Wrzesień, M. and Cwener, A. (2014): Pollination and floral biology of Adonis vernalis L. (Ranunculaceae) - a case study of threatened species. - Acta Soc. Bot. Poloniae 83(1): 29-37.

DövÉNYI, Z. (ed.) (2010): Magyarország kistájainak katasztere. II. átd. bőv. kiad. - MTA Földrajztudományi Kutatóintézet, Budapest, 876 pp.

JankowsKa-B£AszCZUK, M. (1988): Morphological-developmental properties as an agent forming spatial structure of Adonis vernalis (L.) populations. - Acta Soc. Bot. Poloniae 57(4): 573-587.

KEARns, C. A. (2002): Flies and flowers: an enduring partnership. - Wings 25(2): 3-8.

KeVAN, P. (2002): Flowers, pollination, and the associated diversity of flies. - Biodiversity 3(4): $16-18$.

MAJER, J. (1977): Katonalegyek - Gömblegyek (Stratiomyidae - Acroceridae). - Magyarország Állatvilága (Fauna Hungariae) 129, XIV/10. Akadémiai Kiadó, Budapest, 75 pp.

MÁTHÉ, Á. (1977): Az Adonis vernalis L. virágzásának számszerủ kifejezése. - Herba Hung. 16(2): $35-43$.

MÉszÁros, T. and Józan, Zs. (2018): Pollinators (Hymenoptera: Aculeata) of Adonis vernalis in Transdanubia (Hungary). - Studia bot. bung. 49(2): 61-71. https://doi.org/10.17110/StudBot.2018.49.2.61

MıнÁlyı, F. (1960): Fúrólegyek - Trypetidae. - Magyarország Állatvilága (Fauna Hungariae), $\mathrm{XV} / 56$. Akadémiai Kiadó, Budapest, $76 \mathrm{pp}$.

MıнÁlyi, F. (1975): Igazi legyek (Muscidae). - Magyarország Állatvilága (Fauna Hungariae) 124, $\mathrm{XV} / 12$. Akadémiai Kiadó, Budapest, $229 \mathrm{pp}$.

Minályi, F. (1979): Fémeslegyek - Húslegyek (Calliphoridae - Sarcophagidae). - Magyarország Állatvilága (Fauna Hungariae) 135, XV/16. Akadémiai Kiadó, Budapest, 152 pp.

Novais, S. M. A., Nunes, C. A., Santos, N. B., D’Amico, A. R., Fernandes, G. W., Quesada, M., Braga, R. F. and Neves, A. C. O. (2016): Effects of a possible pollinator crisis on food crop production in Brazil. - Plos One 11(11): e0167292. https://doi.org/10.1371/journal.pone.0167292

PAT Kó, F. (2017): A rovarok (Insecta) általi beporzás. - Acta Sci. Transylvanica 25(3): 126-132.

SolTÉsz, Z. (2017): A kétszárnyúakhoz (Diptera) kötődő ökoszisztémaszolgáltatások. -Term.véd. Közlem. 23: 80-99. https://doi.org/10.20332/tvk-jnatconserv.2017.23.80

Ssymank, A., Kearns, C. A., Pape, T. and Thompson, F. C. (2008): Pollinating flies (Diptera): a major contribution to plant diversity and agricultural production. - Biodiversity 9(1-2): 86-89. https://doi.org/10.1080/14888386.2008.9712892 
Tóth, S. (1975): Adatok a Tardi-patak völgye Diptera faunájához. - A Herman Ottó Múzeum Évkönyve 13-14: 587-615.

Tótн, S. (1977): Pöszörlegyek - Ablaklegyek (Bombyliidae - Scenopinidae). - Magyarország Állatvilága (Fauna Hungariae) 127, XIV/12. Akadémiai Kiadó, Budapest, 87 pp.

Tótн, S. (2001): A Bakonyvidék zengőlégy faunája (Diptera: Syrphidae). - Bakony term.tud. kut. eredm. 25: 1-448.

Tóth, S. (2011): Magyarország zengőlégy faunája (Diptera: Syrphidae) - e-Acta Nat. Pannon., Suppl. 1: 1-408.

WÉBER, M. (1975): Táncoslegyek (Empididae). - Magyarország Állatvilága (Fauna Hungariae) 121, $\mathrm{XIV} / 13$. Akadémiai Kiadó, Budapest, 220 pp.

(submitted: 08.01.2020, accepted: 16.07.2020) 